\title{
La confianza como elemento clave en las relaciones interempresariales. Una escala de medida
}

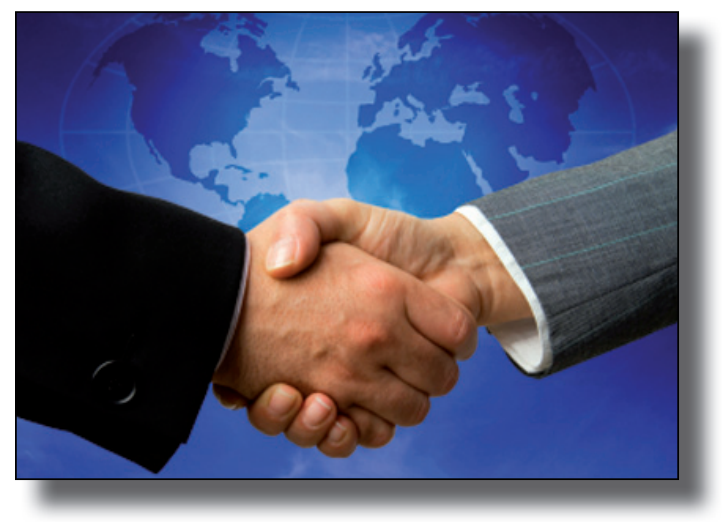

\author{
Manuel Expósito-Langa \\ Dr. en Organización de Empresas \\ Débora Nicolau-Juliá \\ Lcda. en Administración y Dirección de Empresas \\ Josep Capó-Vicedo \\ Dr. en Organización de Empresas
}

UNIVERSITAT POLITÈCNICA DE VALÈNCIA. Dpto. de Organización de Empresas, Pl. Ferrándiz y

Carbonell, 2 - 03801 Alcoy (Alicante). Tfno: +34 966528466 . maexlan@doe.upv.es;

deniju@epsa.upv.es; pepcapo@doe.upv.es

Recibido: 12/11/2010 • Aceptado: 09/05/2011

\section{Trust as a key factor in the enterprise relationships. A scale of measurement}

ABSTRACT
- In recent years, companies in
industrialized countries have
experimented significant
changes in the competitive
context where they move. This
has resulted in reinforcing
certain factors in order to
retain and win new customers
such as trust, accepted as
a key element in economic
exchanges. In this work, and
following a review of the
literature, a scale measuring
confidence, both with both
suppliers and customers, is
proposed and validate to serve
as support for future models.
For this has been chosen as
a study sample the Valencian
textile industry.
- Keywords: trust, confirmatory
factorial analysis, textile
industry.

\section{RESUMEN}

En los últimos años las empresas de países industrializados han experimentado cambios significativos en el contexto competitivo en el que se mueven. Esto les ha llevado a reforzar determinados factores con el objeto de fidelizar y conseguir nuevos clientes, como es el caso de la confianza, aceptada como elemento clave en los intercambios económicos. En este trabajo, y tras una revisión de la literatura, se propone y se valida una escala de medición de la confianza, tanto con proveedores como con clientes, que sirva de apoyo para futuros modelos. Para ello se ha escogido como objeto de estudio la industria textil valenciana.

Palabras Clave: confianza, análisis factorial confirmatorio, industria textil.

\section{INTRODUCCIÓN}

En las dos últimas décadas, los países industrialmente avanzados han experimentado cambios significativos en el contexto competitivo en el que se mueven. Bajo estas circunstancias, las empresas han tratado de ganar clientes al competir no sólo en el precio, sino también en factores como la velocidad de entrega, la calidad del producto y la innovación. Esto ha forzado cambios tanto en la organización interna de las empresas, como en las relaciones interempresariales. En este contexto, el elemento confianza es ampliamente aceptado como un ingrediente clave para el intercambio económico (Beugelsdijk, 2006): es decir, no hay ninguna transacción que no requiera un mínimo de confianza entre las partes. De hecho, para algunos autores, la confianza es la variable universalmente más aceptada como base en cualquier interacción o intercambio humano.

En este trabajo se asumirá la definición de confianza propuesta por Bradach y Eccles (1989) como "la expectativa que libera a un socio del temor de que otro socio actúe de forma oportunista". De esta forma, la confianza constituye uno de los elementos más determinantes del carácter relacional del intercambio.

Por otro lado, no se observa un consenso en la literatura a la hora de establecer una medida unificada de la confianza. Partiendo de esta problemática, en este trabajo se pretende aportar y validar una escala de medida de la confianza que una empresa sostiene con sus proveedores y clientes, que sirva como base para futuros modelos de investigación. 
De esta forma, esta investigación se ha basado en la revisión bibliográfica de anteriores investigaciones de relevancia donde se ha estudiado y medido el concepto de la confianza (ver Anexo I). En la escala resultante se ha valorado tanto el grado de compromiso como su nivel de cumplimiento en las relaciones por parte de proveedores y clientes, así como el interés de éstos en que la relación sea beneficiosa para ambas partes, y la intención por parte de las empresas de mantener o ampliar las relaciones con sus proveedores o clientes.

A continuación, se describe tanto el objeto de estudio como el proceso seguido para la validación de la escala y las conclusiones obtenidas.

\section{TRABAJO EMPÍRICO}

Para llevar a cabo el trabajo de campo se ha elegido la industria textil, un sector heterogéneo y diverso, que comprende un amplio número de actividades productivas, e integrado principalmente por PYMEs de origen familiar. Se trata de un sector considerado de demanda débil y contenido tecnológico bajo, y viene caracterizado por un proceso productivo intensivo en mano de obra. Este trabajo se centra de forma particular en la industria textil valenciana, la cual se encuentra situada principalmente en las comarcas de L'Alcoià, El Comtat y L'Alt Vinalopó en Alicante y La Vall d'Albaida en Valencia. En estas comarcas se generó durante 2008 el $17 \%$ de la producción textil en España (1.825 millones de $€$ ), con una ocupación directa de algo más de 32.000 trabajadores.

Para el estudio empírico se ha considerado el total de la población de empresas textiles identificado a partir de los registros del año 2008 de la base de datos $\mathrm{SABI}^{1}$. El listado inicial de empresas se depuró eliminando aquéllas que bien por su reducido tamaño, o por el tipo de producto, no se consideraron significativas del sector, ya que podían mostrar una excesiva heterogeneidad. A su vez, la información de SABI sirvió para establecer el control de algunas respuestas recogidas.

El trabajo se extendió durante los meses de junio a septiembre de 2009 y la fuente de información básica utilizada fue un cuestionario con escala Likert 1 a 7 . Con anterioridad a su distribución, se realizó un cuestionario piloto que fue completado por 5 empresas seleccionadas del sector en función de su trayectoria e importancia en el mismo para la redefinición y ajuste de las variables e indicadores. El cuestionario final fue formulado tras solicitar también la opinión de un panel de expertos.

Finalmente, la realización del trabajo de campo se desarrolló en base a entrevistas con los directivos de las

\footnotetext{
1 SABI es un directorio de empresas españolas y portuguesas que recoge información general
} y datos financieros. empresas seleccionadas a partir de la encuesta diseñada. A continuación, en la Tabla 1 se muestra la ficha técnica del trabajo:

\begin{tabular}{|c|c|}
\hline UNIVERSO & $\begin{array}{c}\text { Empresas pertenecientes al } \\
\text { sector textil }\end{array}$ \\
\hline ÁMBITO GEOGRÁFICO & Comunidad Valenciana \\
\hline MARCO MUESTRAL & 739 empresas del sector textil \\
\hline ELEMENTO MUESTRAL & $\begin{array}{c}\text { Director de logística/ Director } \\
\text { de operaciones }\end{array}$ \\
\hline TAMAÑO MUESTRAL & $\begin{array}{c}156 \text { empresas del sector textil, } \\
\text { con un indice de respuesta del } \\
21,1 \%\end{array}$ \\
\hline ERROR MUESTRAL & $\begin{array}{c}\text { Nivel de confianza del 95\%, } \\
\text { Z=1,96; } p=q=0,5\end{array}$ \\
\hline DISEÑO MUESTRAL & $\begin{array}{c}\text { Diseño y Pre-tests } \\
\text { cuestionario. Encuesta. }\end{array}$ \\
\hline TRABAJO DE CAMPO & Junio a septiembre de 2009 \\
\hline INFORMACIÓN & $\begin{array}{c}\text { Datos básicos de la empresa, } \\
\text { Confianza con proveedores, } \\
\text { Confianza con clientes }\end{array}$ \\
\hline RECOGIDA &
\end{tabular}

Tabla 1: Ficha técnica de la investigación

\subsection{MEDIDA DE LA CONFIANZA}

Para hacer operativo el concepto de la confianza, se ha intentado plasmar las diferentes visiones identificadas en la bibliografía examinada. De esta forma, se considera importante incorporar la idea de compromiso y relación proveedor-cliente planteada en San Martín, Gutiérrez y Camarero (2004), Ulaga y Eggert (2006), Tian, Lai y Daniel (2008), y Suh y Houston (2010). Así, se propone el ítem 1 (Sus proveedores/clientes clave mantienen habitualmente las promesas realizadas a su empresa). Por otro lado, se encuentran referencias a la obtención de un beneficio compartido en trabajos como Ganesan (1994), Gefen, Kanahanna y Straub (2003), San Martín, Gutiérrez y Camarero (2004), Ulaga y Eggert (2006), Leonidou, Talias y Leonidou, (2008), y Suh y Houston (2010). En esta línea, se propone el ítem 2 (A la hora de tomar decisiones importantes, sus proveedores/clientes clave tienen interés en que la relación sea beneficiosa para ambas partes). Adicionalmente, se ha observado que en algunos trabajos aparece la idea de la satisfacción que se tiene respecto al proveedor y al cliente, por ejemplo en Morgan y Hunt (1994), Ganesan (1994), Gefen, Kanahanna y Straub (2003), San Martín, Gutiérrez y Camarero (2004), Johnston, McCutheon, Stuart et al. (2004) y Ulaga y Eggert (2006), donde proponen una variable que mide directamente "la confianza". Siguiendo estos trabajos se plantea el ítem 3 (Sus proveedores/clientes clave son de confianza). A partir de la idea de la dependencia entre 
empresas a largo plazo planteada en los trabajos de Ganesan (1994), San Martín, Gutiérrez y Camarero (2004) y Ulaga y Eggert (2006), se propone el ítem 4 (Su empresa está muy comprometida en sus relaciones con sus proveedores/ clientes clave). A continuación, y haciendo referencia a la estabilidad de las relaciones mantenidas en la actualidad por parte de la empresa, como se identifica en los trabajos de San Martín (2003), Johnston, McCutheon, Stuart et al. (2004), Ulaga y Eggert (2006) y Suh y Houston (2010), se propone el ítem 5 ( $\mathrm{Su}$ empresa tiene la intención de mantener o ampliar su relación con sus proveedores/clientes clave). Por último, y asociado con la consideración de bienestar de la relación establecida, y siguiendo la línea de trabajos como San Martín (2003) y Ulaga y Eggert (2006), el ítem 6 propone la siguiente pregunta (Su relación con proveedores/ clientes clave va más allá del mero negocio, llegando a ser de amistad o familiar).

A continuación se muestra la Tabla 2 con los ítems planteados. Se utilizará una escala Likert de 1 a 7 , donde $1=$ nunca o totalmente en desacuerdo y $7=$ siempre o totalmente de acuerdo.

\begin{tabular}{|c|}
\hline Confianza con Proveedores \\
\hline $\begin{array}{l}\text { 1. Sus proveedores clave mantienen habitualmente las } \\
\text { promesas realizadas a su empresa. }\end{array}$ \\
\hline $\begin{array}{l}\text { 2. A la hora de tomar decisiones importantes, sus } \\
\text { proveedores clave tienen interés en que la relación sea } \\
\text { beneficiosa para ambas partes. }\end{array}$ \\
\hline 3. Sus proveedores clave son de confianza \\
\hline $\begin{array}{l}\text { 4. Su empresa está muy comprometida en sus relaciones } \\
\text { con sus proveedores clave }\end{array}$ \\
\hline $\begin{array}{l}\text { 5. Su empresa tiene la intención de mantener o ampliar su } \\
\text { relación con sus proveedores clave }\end{array}$ \\
\hline $\begin{array}{l}\text { 6. Su relación con proveedores clave va más allá del mero } \\
\text { negocio, llegando a ser de amistad o familiar }\end{array}$ \\
\hline Confianza con Clientes \\
\hline $\begin{array}{l}\text { 1. Sus clientes clave mantienen habitualmente las } \\
\text { promesas realizadas a su empresa. }\end{array}$ \\
\hline $\begin{array}{l}\text { 2. A la hora de tomar decisiones importantes, sus clientes } \\
\text { clave tienen interés en que la relación sea beneficiosa } \\
\text { para ambas partes. }\end{array}$ \\
\hline 3. Sus clientes clave son de confianza \\
\hline $\begin{array}{l}\text { 4. Su empresa está muy comprometida en sus relaciones } \\
\text { con sus clientes clave }\end{array}$ \\
\hline $\begin{array}{l}\text { 5. Su empresa tiene la intención de mantener o ampliar su } \\
\text { relación con sus clientes clave }\end{array}$ \\
\hline $\begin{array}{l}\text { 6. Su relación con clientes clave va más allá del mero } \\
\text { negocio, llegando a ser de amistad o familiar }\end{array}$ \\
\hline
\end{tabular}

Tabla 2: Items utilizados en la medida de la confianza con proveedores y clientes

\section{RESULTADOS}

La validez de una escala hace referencia al grado en el cual el proceso de medida está libre tanto de errores sistemáticos como de errores aleatorios (Kinnear y Taylor, 1995). La creación de una escala exige un proceso de contrastación de las propiedades psicométricas del instrumento de medida. De esta forma, se ha desarrollado un análisis factorial confirmatorio que especifique las relaciones de las medidas observadas. Las pruebas se han realizado con el software EQS v. 6.1.

Previamente a la realización de las pruebas necesarias para validar una escala, tal y como señala Bollen (1989), hay que asegurar la validez de contenido de la misma. Ésta constituye una evaluación subjetiva que depende del investigador, por lo que se observa que no existe un criterio objetivo. En el presente caso las escalas han sido desarrolladas siguiendo la metodología habitual del examen de investigaciones previas cuyas propiedades han sido testadas a lo largo de la literatura, lo que confirmaría dicha validez de contenido.

A continuación, con el objeto de depurar y validar las escalas utilizadas, se ha aplicado el desarrollo de modelos estructurales (Hair, Anderson y Tatham, 1999), realizando las pruebas pertinentes para medir su consistencia. Este análisis de los datos se lleva a cabo mediante el análisis factorial confirmatorio, donde se observará si se cumple la validez convergente, lo que garantiza la cualidad unidimensional de los ítems, es decir, que cada ítem contribuye a la formación de un solo factor. A continuación se describen aquellos parámetros, así como el valor adecuado que deberían obtener en el análisis para poder asegurar la validez del constructo.

En primer lugar hay que observar las cargas factoriales de los ítems $(\lambda)$, que no deberían ser inferiores a $(0,7)$. Otro valor de interés viene dado a través del indicador de consistencia interna de la escala, medido a través de la fiabilidad compuesta $\left(\rho_{c}\right)$, y cuyo valor es recomendable que sea superior a $(0,7)$ (Fornell y Larcker, 1981), y el estadístico Alpha de Cronbach, también con un valor recomendado de $(0,7)$. Otra medida a tener en cuenta es el indicador de la Varianza Extraída (AVE), donde la escala deberá presentar un valor superior a $(0,5)$.

Por otro lado es interesante analizar los valores asociados a la bondad del ajuste del modelo. Tal y como recomiendan algunos autores (Bollen, 1989) es recomendable utilizar diferentes medidas para esta comprobación. En este caso se ha prestado especial atención a los siguientes:

- El estadístico Chi-cuadrado indica si el modelo se ajusta a los datos ( $\mathrm{p}$-valor $>0,05)$.

- $\quad$ El índice de bondad de ajuste GFI (Goodness of Fit Index) mide la variabilidad explicada por el modelo $(>0,9)$.

- $\quad$ El índice de bondad ajustado $A G F$ (Adjusted Goodness of Fit index) ajusta el índice anterior por los grados de libertad $(>0,9)$

- $\quad$ El coeficiente CFI (Comparative Fix Index) indica en qué grado es mejor el ajuste del modelo en comparación con un modelo de referencia $(>0,9)$. 
- $\quad$ Por último, el RMSEA (Root Mean Square Error of Approximation) $(<0,05)$.

A continuación se discuten los diferentes AFC realizados sobre la escala de la confianza, con el fin de validarla con los datos que se han obtenido en la muestra de empresas seleccionada.

\begin{tabular}{|c|c|c|c|c|}
\hline & \multicolumn{2}{|c|}{ Confianza con proveedores } & \multicolumn{2}{|c|}{ Confianza con clientes } \\
\hline Análisis factorial con 6 ítems & $\begin{array}{l}\text { Carga } \\
\text { factorial } \\
(\lambda)\end{array}$ & Fiabilidad & $\begin{array}{l}\text { Carga } \\
\text { factorial } \\
(\lambda)\end{array}$ & Fiabilidad \\
\hline $\begin{array}{l}\text { 1.Sus proveedores/clientes clave } \\
\text { mantienen habitualmente las } \\
\text { promesas realizadas a su empresa }\end{array}$ &, 749 & \multirow{6}{*}{$\begin{array}{c}X^{2}=15,150 \\
\text { p-valor }=, 087 \\
\text { GFI }=, 970 \\
A G F=, 929 \\
C F I=, 987 \\
E A=, 066(, 000-, 122) \\
\text { de Cronbach }=, 859 \\
\rho_{c}=, 885 \\
\text { AVE }=, 567\end{array}$} &, 738 & \multirow{6}{*}{$\begin{array}{c}X^{2}=20,423 \\
\mathrm{p}-\mathrm{valor}=, 015 \\
\mathrm{GFI}=, 964 \\
\mathrm{AGF}=, 917 \\
\mathrm{CFI}=, 977 \\
\text { RMSEA }=, 090(, 037-, 143) \\
\alpha \text { de Cronbach }=, 863 \\
\rho_{\mathrm{c}}=, 887 \\
\text { AVE }=, 574\end{array}$} \\
\hline $\begin{array}{l}\text { 2.A la hora de tomar decisiones } \\
\text { importantes, sus proveedores/clientes } \\
\text { clave tienen interés en que la } \\
\text { relación sea beneficiosa para ambas } \\
\text { partes }\end{array}$ & 811 & &, 733 & \\
\hline $\begin{array}{l}\text { 3.Sus proveedores/clientes clave son de } \\
\text { confianza }\end{array}$ &, 751 & & 858 & \\
\hline $\begin{array}{l}\text { 4.Su empresa está muy comprometida } \\
\text { en sus relaciones con sus } \\
\text { proveedores/clientes clave }\end{array}$ &, 854 & & 873 & \\
\hline $\begin{array}{l}\text { 5.Su empresa tiene la intención de } \\
\text { mantener o ampliar su relación con } \\
\text { sus proveedores/clientes clave }\end{array}$ & ,797 & &, 802 & \\
\hline $\begin{array}{l}\text { 6.Su relación con proveedores/clientes } \\
\text { clave va más allá del mero negocio, } \\
\text { llegando a ser amistad o familiar }\end{array}$ &, 508 & &, 473 & \\
\hline
\end{tabular}

Tabla 3: Análisis factorial de las seis actividades de confianza e indicadores de fiabilidad

\begin{tabular}{|c|c|c|c|c|}
\hline & \multicolumn{2}{|c|}{ Confianza con proveedores } & \multicolumn{2}{|c|}{ Confianza con clientes } \\
\hline Análisis factorial con 5 ítems & $\begin{array}{c}\text { Carga } \\
\text { factorial } \\
(\lambda)\end{array}$ & Fiabilidad & $\begin{array}{c}\text { Carga } \\
\text { factorial } \\
(\lambda)\end{array}$ & Fiabilidad \\
\hline $\begin{array}{l}\text { 1.Sus proveedores/clientes clave } \\
\text { mantienen habitualmente las } \\
\text { promesas realizadas a su empresa }\end{array}$ & ,743 & \multirow{5}{*}{$\begin{array}{c}X^{2}=11,422 \\
\text { p-valor }=, 044 \\
\text { GFI }=, 972 \\
\mathrm{AGF}=, 917 \\
\mathrm{CFI}=, 985 \\
\mathrm{SEA}=, 091(, 014-, 161) \\
\text { de Cronbach }=, 892 \\
\rho \mathrm{c}=, 894 \\
\mathrm{AVE}=, 629\end{array}$} & ,733 & \multirow{5}{*}{$\begin{array}{c}X^{2}=9,220 \\
\text { p-valor }=, 101 \\
\text { GFI }=, 979 \\
\text { AGF }=, 937 \\
\text { CFI }=, 991 \\
\text { RMSEA }=, 074(, 000-, 147) \\
\alpha \text { de Cronbach }=, 895 \\
\rho c=, 900 \\
\text { AVE }=, 645\end{array}$} \\
\hline $\begin{array}{l}\text { 2.A la hora de tomar decisiones } \\
\text { importantes, sus proveedores/clientes } \\
\text { clave tienen interés en que la } \\
\text { relación sea beneficiosa para ambas } \\
\text { partes }\end{array}$ & 815 & & ,733 & \\
\hline $\begin{array}{l}\text { 3.Sus proveedores/clientes clave son de } \\
\text { confianza }\end{array}$ & ,756 & &, 851 & \\
\hline $\begin{array}{l}\text { 4.Su empresa está muy comprometida } \\
\text { en sus relaciones con sus } \\
\text { proveedores/clientes clave }\end{array}$ &, 854 & &, 881 & \\
\hline $\begin{array}{l}\text { 5.Su empresa tiene la intención de } \\
\text { mantener o ampliar su relación con } \\
\text { sus proveedores/clientes clave }\end{array}$ & ,794 & & 805 & \\
\hline
\end{tabular}

Tabla 4: Análisis factorial de las cinco actividades de confianza e indicadores de fiabilidad 
La Tabla 3 muestra el primer AFC, donde se puede comprobar que el último ítem (6. Su relación con proveedores/clientes clave va más allá del mero negocio, llegando a ser amistad o familiar) tiene cargas factoriales con valores inferiores a $(0,7)$ en ambas escalas. Por otro lado, la Chi-cuadrado, tiene un p-valor cercano a $(0,05)$, superándolo en el caso de los proveedores, mientras que los indicadores GFI, AGF y CFI tienen valores superiores a $(0,9)$, como es deseable. El RMSEA de los proveedores contiene el valor cero en el intervalo, y en los clientes su valor es próximo. Tanto los valores del Alpha de Cronbach como la fiabilidad compuesta para los proveedores como para los clientes, son aceptables. Por último, el análisis de la varianza extraída (AVE) da valores superiores a $(0,5)$. A partir de estos resultados, es interesante desarrollar un nuevo AFC eliminando el ítem 6 de la escala.

Este nuevo AFC aparece en la Tabla 4, pudiéndose comprobar que todos los ítems tienen cargas factoriales superiores a $(0,7)$ y que el p-valor de la Chi-cuadrado es cercano a $(0,05)$ en el caso de la escala de clientes, y mayor para proveedores. Por lo que respecta a los indicadores GFI, AGF y CFI, todos tienen valores superiores a $(0,9)$ tanto en el caso de proveedores como de clientes. En cuanto al indicador RMSEA de proveedores, se puede comprobar que el cero está muy próximo al intervalo, mientras que en el caso de los clientes está incluido en el mismo. Los valores de Alpha de Cronbach y de fiabilidad compuesta son superiores a $(0,7)$. Por último, el AVE es superior a $(0,5)$ en ambos casos. Al ser todos los valores aceptables, se puede concluir que estos cinco ítems integrarían una escala validada para la confianza.

\section{CONCLUSIONES}

Mediante este trabajo se ha pretendido contribuir a la literatura sobre el concepto de la confianza mediante una propuesta y validación de dos escalas de medida, una para proveedores y otra para clientes. Para cumplir con el objetivo propuesto se ha llevado a cabo una revisión bibliográfica de aquellos trabajos considerados como relevantes. A continuación se ha sintetizado una escala de medida para proveedores y clientes, integrada inicialmente por 6 ítems, lo que ha permitido una mejor comprensión del concepto y que ha sido necesario depurar mediante sucesivos análisis factoriales confirmatorios.

El resultado de este análisis ha proporcionado apoyo empírico a otras investigaciones realizadas sobre la confianza, como por ejemplo Morgan y Hunt (1994), Ganesan (1994), San Martín, Gutiérrez y Camarero (2004), Ulaga y Eggert (2006), Tian, Lai y Daniel (2008), Leonidou, Talías y Leonidu (2008) y Suh y Houston (2010), entre otros. Sin embargo, la escala final, tanto para proveedores como para clientes, ha sido validada únicamente con 5 de los 6 ítems. Los ítems definitivos han sido: mantenimiento de las promesas; interés en que la relación sea beneficiosa para ambas partes; ser de confianza; compromiso con la relación e intención de mantener y ampliar las relaciones.

Sin embargo, un ítem no ha sido contemplado en la escala final (relación más allá del mero negocio, llegando a ser de amistad o familiar). Se ha considerado que por su particularidad puede no haber sido valorado adecuadamente por parte de los encuestados. Si se compara con el resto de ítems, éstos implican el desarrollo de relaciones más formales, y tal vez ésta sea la causa que ha llevado a que el ítem no haya sido adecuadamente percibido por los encuestados, y, por tanto, no cumpla los requisitos para formar parte de la escala de la confianza.

En definitiva, se puede considerar que el objetivo propuesto en este trabajo de investigación ha sido cubierto mediante la depuración final de las escalas. Cabe apuntar que este resultado es un primer paso para continuar con investigaciones futuras, donde se propondrán modelos teóricos que permitan estudiar la influencia de la confianza sobre otras variables basadas en la integración entre empresas, y su posible efecto sobre el resultado empresarial.

El trabajo cuenta a su vez con algunas limitaciones que cabe señalar. Así, por ejemplo, la confianza se ha medido en un momento puntual, pero hay que tener en consideración que es el resultado del transcurso de relaciones desarrolladas a lo largo del tiempo, por lo que en el futuro será interesante plantear un análisis longitudinal. Cabe a su vez plantear la validación de la escala en otros sectores industriales, quizá no tan concentrados geográficamente como el textil valenciano. Esto permitiría observar si las escalas son robustas en otros contextos. En este caso, se debería desarrollar un proceso de invarianza métrica para comprobar si los resultados son consistentes en ambos grupos y si los conceptos que se miden se interpretan de la misma manera.

\section{AGRADECIMIENTOS}

Esta investigación ha sido financiada por el Ministerio de Educación y Ciencia, Plan Nacional de I+D+i (2007-2010), proyecto "El Distrito Industrial: el impacto del Capital Social sobre la Gestión de la Cadena de Suministro" (SEJ200762876/ECON). 


\section{ANEXO I. TRABAJOS RELATIVOS A LA CONFIANZA}

\begin{tabular}{|c|c|c|c|}
\hline Autor/es & Muestra & $\begin{array}{l}\text { Escala de la } \\
\text { Confianza }\end{array}$ & Ítems \\
\hline $\begin{array}{l}\text { Morgan y } \\
\text { Hunt (1994) }\end{array}$ & $\begin{array}{l}\text { Minoristas de } \\
\text { neumáticos de } \\
\text { vehículos }\end{array}$ & $\begin{array}{l}\text { Validada con un } \\
\text { total de } 3 \text { ítems. }\end{array}$ & $\begin{array}{l}\text { 1. No se puede confiar en ocasiones, } 2 \text {. Se puede contar para hacer lo } \\
\text { correcto, 3. Tiene una alta integridad }\end{array}$ \\
\hline $\begin{array}{l}\text { Ganesan } \\
(1994)\end{array}$ & $\begin{array}{l}\text { Proveedores y } \\
\text { minoristas y sus } \\
\text { proveedores }\end{array}$ & $\begin{array}{l}\text { Creación propia. La } \\
\text { escala de confianza } \\
\text { del cliente con el } \\
\text { proveedor se ha } \\
\text { validado con un } \\
\text { total de } 5 \text { ítems y la } \\
\text { escala de confianza } \\
\text { del proveedor en el } \\
\text { cliente con un total } \\
\text { de } 3 \text { ítems }\end{array}$ & $\begin{array}{l}\text { Cliente con Proveedor: } \\
\text { 1. Este proveedor se ha esforzado por nosotros en el pasado, 2. Este } \\
\text { proveedor se preocupa por nosotros, 3. En tiempos de escasez este } \\
\text { proveedor se ha portado bien con nosotros, } 4 \text {. Este proveedor es como } \\
\text { un amigo, 5. Creemos que este proveedor ha estado siempre a nuestro } \\
\text { lado. } \\
\text { Proveedor con cliente: } \\
\text { 1. Este cliente ha hecho esfuerzos por nosotros en el pasado, 2. El } \\
\text { cliente se preocupa por mi bienestar, 3. En tiempos con problemas de } \\
\text { entrega este cliente ha sido muy comprensivo. }\end{array}$ \\
\hline $\begin{array}{l}\text { Doney y } \\
\text { Cannon } \\
(1997)\end{array}$ & $\begin{array}{l}\text { Datos de compra } \\
\text { de las empresas } \\
\text { dedicadas a } \\
\text { la fabricación } \\
\text { industrial }\end{array}$ & $\begin{array}{l}\text { A partir del trabajo } \\
\text { de Ganesan (1994) } \\
\text { y creación propia. } \\
\text { Validada con un } \\
\text { total de } 7 \text { ítems. }\end{array}$ & $\begin{array}{l}\text { 1. Este vendedor ha fracasado con el trabajo realizado con nosotros, } \\
\text { 2. Este vendedor no hace afirmaciones falsas, } 3 \text {. Creemos que este } \\
\text { vendedor no se abre totalmente al trato con nosotros, } 4 \text {. Este vendedor } \\
\text { solo está preocupado por sí mismo, } 5 \text {. Este vendedor no parece } \\
\text { preocuparse por nuestras necesidades, } 6 \text {. La gente de la empresa no se } \\
\text { fía de este vendedor, } 7 \text {. Este vendedor no es digno de confianza. }\end{array}$ \\
\hline $\begin{array}{l}\text { Gefen, } \\
\text { Karahanna } \\
\text { y Straub } \\
(2003)\end{array}$ & $\begin{array}{l}\text { Datos de } \\
\text { visitantes y su } \\
\text { intención de } \\
\text { compra en un } \\
\text { sitio web }\end{array}$ & $\begin{array}{l}\text { A partir de los } \\
\text { trabajos de Ganesan } \\
\text { (1994) y Doney y } \\
\text { Cannon (1997). } \\
\text { Validada con un } \\
\text { total de } 7 \text { items. }\end{array}$ & $\begin{array}{l}\text { 1. En base a mi experiencia con vendedores anteriores, sé que este es } \\
\text { honesto, } 2 \text {. En base a mi experiencia con vendedores anteriores, sé } \\
\text { que este se preocupa por los clientes, } 3 \text {. En base a mi experiencia con } \\
\text { vendedores en el pasado se que este no es oportunista, } 4 \text {. En base con } \\
\text { mi experiencia con vendedores en el pasado, se que este ofrece un buen } \\
\text { servicio, 5. Basándome en experiencias anteriores con vendedores, este } \\
\text { es previsible, } 6 \text {. Basándome en experiencias con proveedores anteriores, } \\
\text { sé que este es digno de confianza, } 7 \text {. Basándome en experiencias } \\
\text { anteriores se nota que este vendedor conoce su mercado. }\end{array}$ \\
\hline $\begin{array}{l}\text { Johnston, } \\
\text { McCutcheon, } \\
\text { Stuart et al. } \\
(2004)\end{array}$ & $\begin{array}{l}\text { Miembros de } \\
\text { la asociación } \\
\text { de gerentes } \\
\text { de compra de } \\
\text { Canadá }\end{array}$ & $\begin{array}{l}\text { A partir de los } \\
\text { trabajos de Ganesan } \\
\text { (1994) y Doney y } \\
\text { Cannon (1997). } \\
\text { Validada con un } \\
\text { total de } 4 \text { items. }\end{array}$ & $\begin{array}{l}\text { 1. Tenemos gran confianza personal en la otra parte, } 2 \text {. Tenemos } \\
\text { confianza en que se establezca un negocio fuerte con la otra empresa, } \\
\text { 3. Siempre podemos confiar el uno con el otro, } 4 \text {. Creo que ha trabajado } \\
\text { duro para mantener en el futuro una relación con mi empresa. }\end{array}$ \\
\hline $\begin{array}{l}\text { San Martín, } \\
\text { Gutiérrez y } \\
\text { Camarero } \\
(2004)\end{array}$ & $\begin{array}{l}\text { Directivos de } \\
\text { los servicios } \\
\text { de garajes y } \\
\text { sus clientes } \\
\text { habituales }\end{array}$ & $\begin{array}{l}\text { A partir de los } \\
\text { trabajos de Morgan } \\
\text { y Hunt (1994), } \\
\text { Doney y Cannon } \\
\text { (1997) y Ganesan } \\
\text { (1994). Validada con } \\
\text { un total de } 8 \text { items }\end{array}$ & $\begin{array}{l}\text { 1. Confianza en el garaje } X \text { con competencia profesional, 2. Creencia } \\
\text { que el garaje } X \text { tiene un personal altamente cualificado, 3. La confianza } \\
\text { en las buenas intenciones que tienen el garaje } X, 4 \text {. La creencia de que } \\
\text { el garaje } X \text { cumplirá sus promesas, } 5 \text {. Las preocupaciones del garaje } \\
X \text { acerca de la satisfacción del cliente, } 6 \text {. EL garaje } X \text { se esfuerza por } \\
\text { dar una buena atención personal, } 7 \text {. El garaje } X \text { ofrece información } \\
\text { detallada a sus clientes sin que la soliciten, } 8 \text {. El estilo del trabajo del } \\
\text { garaje } X \text { es el que más me gusta. }\end{array}$ \\
\hline $\begin{array}{l}\text { Ulaga y } \\
\text { Eggert (2006) }\end{array}$ & $\begin{array}{l}\text { Fabricantes } \\
\text { químicos, } \\
\text { mecánicos y } \\
\text { de la industria } \\
\text { eléctrica }\end{array}$ & $\begin{array}{l}\text { A partir de los } \\
\text { trabajos de Sullivan } \\
\text { y Peterson (1982), } \\
\text { Morgan y Hunt } \\
\text { (1994), Ganesan } \\
\text { (1994) y Doney y } \\
\text { Cannon (1997). } \\
\text { Validada con un } \\
\text { total de } 5 \text { items. }\end{array}$ & $\begin{array}{l}\text { 1. El proveedor principal mantiene la promesa que hace a nuestra } \\
\text { empresa, 2. El proveedor principal se preocupa de que nuestro negocio } \\
\text { tenga éxito, 3. El proveedor principal considera nuestro bienestar así } \\
\text { como el suyo propio a la hora de tomar decisiones importantes, } 4 \text {. La } \\
\text { empresa confía en que el proveedor principal siempre tiene los intereses } \\
\text { de la misma en mente, 5. El proveedor principal es digno de confianza. }\end{array}$ \\
\hline
\end{tabular}




\begin{tabular}{|c|c|c|c|}
\hline $\begin{array}{l}\text { Tian, Lai y } \\
\text { Daniel (2008) }\end{array}$ & $\begin{array}{l}\text { Gerentes de } \\
\text { empresas de } \\
\text { diferentes } \\
\text { industrias }\end{array}$ & $\begin{array}{l}\text { Adaptado del } \\
\text { trabajo de Johnston, } \\
\text { McCutcheon, } \\
\text { Stuart et al (2004). } \\
\text { Validada con un } \\
\text { total de } 3 \text { ítems. }\end{array}$ & $\begin{array}{l}\text { 1. Por lo general las empresas mantiene las promesas que nos hacen, } 2 . \\
\text { Están preocupados por el bienestar de nuestra empresa, y nunca hacen } \\
\text { nada por herir el mismo, 3. La compañía tiene una conducta ética }\end{array}$ \\
\hline $\begin{array}{l}\text { Leonidou, } \\
\text { Talias y } \\
\text { Leonidou } \\
\text { (2008) }\end{array}$ & $\begin{array}{l}\text { Empresas } \\
\text { estadounidenses } \\
\text { exportadoras al } \\
\text { extranjero }\end{array}$ & $\begin{array}{l}\text { A partir de los } \\
\text { trabajos de Morgan } \\
\text { y Hunt (1994) y } \\
\text { Doney y Cannon } \\
\text { (1997) }\end{array}$ & $\begin{array}{l}\text { 1. Confían unos en otros para mantener un secreto comercial } \\
\text { relativo a la relación de trabajo, } 2 \text {. Sospechan unos de otros por un } \\
\text { comportamiento caracterizado por el engaño y/o el fraude. }\end{array}$ \\
\hline $\begin{array}{l}\text { Suh y } \\
\text { Houston } \\
(2010)\end{array}$ & $\begin{array}{l}201 \text { empresas } \\
\text { compradoras } \\
\text { elegidas al azar } \\
\text { de una base de } \\
\text { datos }\end{array}$ & $\begin{array}{l}\text { A partir de los } \\
\text { trabajos de Morgan } \\
\text { y Hunt (1994), } \\
\text { Ganesan (1994), } \\
\text { y Doney y Cannon } \\
\text { (1997). Validada con } \\
\text { un total de } 10 \text { ítems } \\
\text { (5 para la parte de } \\
\text { benevolencia, y } 5 \\
\text { más para la parte de } \\
\text { integridad) }\end{array}$ & $\begin{array}{l}\text { Benevolencia: } \\
\text { 1. Aunque cambien las circunstancias, creemos que los socios estarán } \\
\text { en condiciones de ofrecer asistencia y apoyo, 2. El socio se preocupa } \\
\text { por nuestro bienestar cuando se toman decisiones importantes, } 3 . \\
\text { Cuando compartimos los problemas, sabemos que van a responder } \\
\text { con comprensión, } 4 \text {. Podemos contar que en el futuro las decisiones } \\
\text { tomadas por el socio no nos afectarán, } 5 \text {. Cuando tratamos cosas } \\
\text { importantes, podemos contar con el apoyo del socio. } \\
\text { Integridad: } \\
\text { 1. Aún cuando nuestro socio nos da una explicación poco probable, } \\
\text { estamos seguros de que dice la verdad, 2. El socio nos informa de } \\
\text { aquello que resulta inexacto, } 3 \text {. El socio mantiene las promesas que } \\
\text { nos hace, 4. Cuando el socio nos da algún consejo, sabemos que está } \\
\text { queriendo ayudarnos, } 5 \text {. Contamos con nuestro socio totalmente. }\end{array}$ \\
\hline
\end{tabular}

\section{BIBLIOGRAFÍA}

- Beugelsdijk S. "A note on the theory and measurement of trust in explaining differences in economic growth". Cambridge Journal of Economics. 2006, Vol.30, p. 371387.

- Bradach JL, Eccles RG. "Price, authority, and trust: From ideal types to plural forms". Annual Review of Sociology. 1989, Vol. 15, p. 97-118.

- Bollen KA. Structural equations with latent variables. New York: John Wiley \& Sons, 1989.

- Doney PM, Cannon JP. "An examination of the nature of trust in buyer-seller relationships". Journal of Marketing. 1997, Vol. 61- 2, p. 35-51.

- Fornell C, Larcker DF. "Evaluating structural equations models with unobservable variables and measurement error". Journal of Marketing Research. 1981, Vol. 18, p. 39-50.

- Ganesan S. "Determinants of long-term orientation in buyer-seller relationships". Journal of Marketing. 1994, Vol. 58-2, p. 1-19.

- Gefen D, Kanahanna E, Straub DW. "Trust and tam in online shopping: an integrated model." MIS Quartely. 2003, Vol. 27-1, p. 51-90.

- Hair JF, Anderson RE, Tatham RL, et al. Análisis Multivariante. Madrid: Ed. Prentice Hall, 1999.

- Johnston DA, McCutcheon DM, Stuart, Fl, Kerwood $\mathrm{H}$. "Effects of supplier trust on performance of cooperative supplier relationships". Journal of Operations Management. 2004, Vol. 22-1, p. 23-38.

- Kinnear JC. Taylor JR. Investigación de mercados: un enfoque aplicado. Madrid: Ed. McGraw Hill, 1995.

- Leonidou LC, Talias MA, Leonidou CN. "Exercised power as a driver of trust and commitment in crossborder industrial buyer-seller relationships". Industrial Marketing Management. 2008, Vol. 37, p. 92-103.

- Morgan RM, Hunt SD. "The commitment-trust theory of relationship marketing". Journal of Marketing. 1994, Vol 58-3, p. 20-38.

- San Martín S, Gutiérrez J, Camarero C. "The consumer's relational commitment: main dimensions and antecedents". Journal of Retailing and Consumer Services. 2004, Vol. 11, p. 351-367.

- Suh T, Houston MB. "Distinguishing supplier reputation from trust in buyer-supplier relationships". Industrial Marketing Management. 2010, Vol. 39, p. 744-751.

- Tian Y, Lai F, Daniel F. "An examination of the nature of trust in logistics outsourcing relationship Empirical evidence from China". Industrial Management \& Data Systems. 2008, Vol. 108-3, p. 346-367.

- Ulaga W, Eggert A. (2006). "Relationship value and relationship quality, broadening the nomological network of business-to-business relationship". European Journal of Marketing. 2006, Vol. 40-3/4, p. 311-327. 